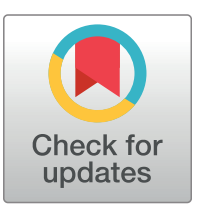

\section{OPENACCESS}

Citation: Qin J, Mai Y, Li Y, Jiang Z, Gao Y (2017) Effect of mild hypothermia preconditioning against low temperature $\left(4^{\circ} \mathrm{C}\right)$ induced rat liver cell injury in vitro. PLoS ONE 12(4): e0176652. https://doi.org/ 10.1371/journal.pone.0176652

Editor: Beicheng Sun, The First Affiliated Hospital of Nanjing Medical University, CHINA

Received: January 1, 2017

Accepted: April 13, 2017

Published: April 28, 2017

Copyright: @ 2017 Qin et al. This is an open access article distributed under the terms of the Creative Commons Attribution License, which permits unrestricted use, distribution, and reproduction in any medium, provided the original author and source are credited.

Data Availability Statement: All relevant data are within the paper and its Supporting Information files.

Funding: This study was supported by the grants from the National High Technology Research and Development Program of China ("863" Program 2012AA020505); National Natural Science Foundation of China (81470875); Science and Technology Planning Project of Guangdong Province (2014B020227002,2015B020229002, 2015B090903069); Natural Science Foundation of Guangdong Province (2014A030312013);
RESEARCH ARTICLE

\section{Effect of mild hypothermia preconditioning against low temperature $\left(4^{\circ} \mathrm{C}\right)$ induced rat liver cell injury in vitro}

\author{
Jiasheng Qin ${ }^{1,2 \odot}$, Yanxing Mai ${ }^{3 \odot}$, Yang $\mathrm{Li}^{1,2}$, Zesheng Jiang ${ }^{1,2}, \mathrm{Yi} \mathrm{Gao}^{1,2 *}$
}

1 Second Department of Hepatobiliary Surgery, Zhujiang Hospital, Southern Medical University, Guangzhou, Guangdong, P.R. China, 2 Institute of Regenerative Medicine, Southern Medical University, Guangzhou, Guangdong, P.R. China, 3 Department of Geriatrics, Zhujiang Hospital, Southern Medical University, Guangzhou, Guangdong, P.R. China

๑ These authors contributed equally to this work.

* gaoyi6146@163.com

\section{Abstract}

Bioartificial liver holds special position in the field of regenerative medicine, and cold environment at $4^{\circ} \mathrm{C}$ is widely used for the short storage of both organ and liver cell for later application. However, the disadvantages of such cold storage could influence cell viability and lead to cell apoptosis in different degrees. In this study, we mainly explore the pre-protective effect of mild hypothermia against low temperature $\left(4^{\circ} \mathrm{C}\right)$-induced rat liver cell injury in vitro. Our results indicated that the precondition with mild hypothermia could increase cell viability, such as cell proliferation, LDH regulation and glycogen synthesis ability of liver cell. The precondition also decreased the ROS production and relieved cell apoptosis in liver cells. Compared with the model group, the mitochondrial membrane potential was restored in the mild hypothermia group, as well as the mitochondrial membrane permeability transition pore opening, indicating that the therapeutic mechanism was related to mitochondrial protection. Further analysis showed that PI3K-Akt-GSK3 $\beta$ signal pathway might be associated with the pre-protective effect of mild hypothermia. Thus, our study suggested that the precondition with mild hypothermia hold the protective effect for liver cell in cold environment, and further developed a novel strategy for the storage of liver seed cells, even bioartificial liver.

\section{Introduction}

With the development of regenerative medicine, bioartificial liver has attracted scientists' attention in recent years, for its promising application in disease treatment. Somatic liver cells are also widely used for pharmacological and toxicological research, as well as for cell transplantation. In present, cold environment at $2-8^{\circ} \mathrm{C}$ (usually at $4^{\circ} \mathrm{C}$ ) is used for the short storage of both the organ and liver cell for later application, and the storage time can vary from several minutes to several hours for different researches. However, scientists have demonstrated the disadvantages of such cold storage could influence cell viability and lead to oxidative damage, mitochondrial dysfunction and cell apoptosis in different degrees $[1,2]$. Therefore, the 
Educational Commission of Guangdong Province (C10319040).

Competing interests: The authors have declared that no competing interests exist. optimized storage method is essential for the application of bioartificial liver and other bioengineering technologies in clinical studies.

Mild hypothermia has been reported to be a very promising neuroprotective therapeutic strategy for patients with brain injury [3-8]. Some groups also demonstrated that the hypothermia could protect liver cell from cell death or apoptosis in some hepatic diseases [9-11]. In recent years, the clinical application of the hypothermia has showed the therapeutic action for the patients with those diseases, and researchers also try to explain the mechanism for the action [12]. For example, mild hypothermia holds the ability to up-regulate the expression of anti-apoptotic gene Bcl-2, and decrease the levels of some inflammatory chemokines (such as IL-8, MCP-1 and COX-2) in endothelial cells [13]. Some scientists also indicated that hypothermia could induce the expression of cold-inducible RNA-binding protein to inhibit cell apoptosis induced by tumor necrosis factor- $\alpha$ via the activation of extracellular signal-regulated kinase [14]. However, most reports describe the complex mechanism about the effect of mild hypothermia on the brain, the detailed molecular mechanisms of underlying potential beneficial effects of hypothermia treatment on the liver cell injury or liver failure may be still far away from our understanding.

As known, cold storage (usually at $4^{\circ} \mathrm{C}$ ) can lead to oxidative damage and cell apoptosis, and liver cell apoptosis is always regarded as an important component of some liver diseases, and the apoptotic signaling pathways mediated by Fas and other apoptotic genes hold significant potential during the process [15-19]. In 2004, Fu et al first evaluated the hepatocyte apoptosis with the treatment of mild hypothermia, and their research indicated that mild hypothermia $\left(26^{\circ} \mathrm{C}\right)$ could suppress Fas-mediated apoptotic signaling pathways in liver cells. This function mainly depended on the inhibition of some signaling events, such as cytochrome $\mathrm{c}$ release, effector caspase activation, and so on [10]. In recent years, the protective effect of mild hypothermia was also evaluated in some other kinds of liver cell injury models to clarify more detailed mechanisms. Sakurai et al. suggested that hypothermia could protect hepatic cell from cell death through the reduction of ROS production in fulminant hepatitis directly. In their study, concanavalin A-induced hepatitis models were established in mice, and the hypothermia group were kept at $25^{\circ} \mathrm{C}$. Their results indicated that hypothermia treatment hold the ability to attenuated liver injury and prolong survival through the activation of c-Jun $\mathrm{N}$-terminal kinase as well as Akt. Similar to their further study about the function of hypothermia in brain injury, the expression of cold-inducible RNA-binding protein was also up-regulated, leading to the decreased hepatocyte apoptosis in the group with mild hypothermia treatment [11, 14].

Therefore, we can conclude that some researchers have demonstrated the protective effect of mild hypothermia from liver cell apoptosis or injury and explored the associated mechanism preliminarily. However, whether the hypothermia still holds the pre-protective effect against liver cell damage and cell apoptisis is still unclear. In this study, low temperature-induced liver cell injury model was established to evaluate the pre-protective effect of mild hypothermia and further establish a novel strategy for the storage of liver seed cells, even bioartificial liver.

\section{Materials and methods}

This study was carried out in strict accordance with the recommendations in the Guide for the Care and Use of Laboratory Animals of the National Institutes of Health. The protocol was approved by the Institutional Animal Care and Use Committee of Southern Medical University.

\section{Cell culture and treatment}

Rat liver cell line, Brl-3A, was used in our study, which was purchased from the Cell Bank of Type Culture Collection of Chinese Academy of Sciences (Shanghai, China). The cell was 
cultured in DMEM (Hyclone, glucose concentration $=11.1 \mathrm{mM}$ ) supplemented with $10 \%$ FBS (Hyclone), $100 \mathrm{U} / \mathrm{mL}$ penicillin and $0.1 \mathrm{~g} / \mathrm{mL}$ streptomycin (Hyclone) at $37^{\circ} \mathrm{C}$ under $5 \% \mathrm{CO} 2$ condition. Brl-3A cells cultured in 6-well plate were treated with the hypothermia when the cells were $70-80 \%$ confluent, and only $1 \mathrm{ml}$ medium was left in each well to ensure the sufficient heat exchange during the treatment process. Five groups were designed as follow (Fig 1A):

1. Control group: Cells were cultured normally in a humidified atmosphere at $37^{\circ} \mathrm{C}$ with $5 \%$ $\mathrm{CO} 2$;

2. Model group: Cells were treated at $4^{\circ} \mathrm{C}$ for $6 \mathrm{~h}$, and further come back to $37^{\circ} \mathrm{C}$ for another 3 $\mathrm{h}$;

3. Mild hypothermia group: Cells were treated with mild hypothermia preconditioning (cells underwent three cycles of incubation at $26^{\circ} \mathrm{C}$ for $10 \mathrm{~min}$ followed with incubation at $37^{\circ} \mathrm{C}$ for $15 \mathrm{~min}$ as the reference [20]), and the following process was the same as the model group;

4. Mild hypothermia+LY294002: All of the operations were the same as the mild hypothermia group. Differently, the medium was further supplemented with $20 \mu \mathrm{M} \mathrm{LY} 294002$ (PI3K inhibitor);

5. Mild hypothermia+LiCl: All of the operations were the same as the mild hypothermia group. Differently, the medium was further supplemented with $20 \mathrm{mM} \mathrm{LiCl}$ (GSK3 $\beta$ inhibitor).

\section{Cell viability assay}

Cell viability assay was performed using CCK- 8 Kit (Dojindo). Briefly, the cells which were seeded into 96-well plates at a density of $1 \times 10^{4}$ cells/well, were detected with $10 \mu \mathrm{L}$ CCK-8, and incubated at $37^{\circ} \mathrm{C}$ for $4 \mathrm{~h}$. The absorbance was measured at $450 \mathrm{~nm}$. In this study, the proliferation index $=$ the absorbance of experimental group-the absorbance of blank group, was used to evaluate the cell viability of each group. Besides, cell proliferation ability was evaluated with Click-iT® Plus EdU Proliferation Kit (Alexa Fluor® 555, Thermo Fisher Scientific), and the assay was mainly performed according to the manufacturer's instructions.

To further evaluate cell viability, the extracellular/intracellular LDH, intracellular glycogen and glucose in medium in each group were measured with LDH-Cytotoxicity Colorimetric Assay Kitll(BioVision), Glycogen Colorimetric/Fluorometric Assay Kit (BioVision) and Amplex $®$ Red Glucose Assay Kit (BioVision) according to the manufacturer's instruction respectively.

\section{Transmission electron microscope (TEM) assay}

The cell samples were fixed in glutaraldehyde first, and further dehydrated in graded ethyl alcohols, and embedded with Epon812 finally. In this study, the ultrathin section was cut with an Ultracut $\mathrm{E}$ ultramicrotome, and the section was stained with uranyl acetate and lead citrate, and further observed under TEM as previously described [21-23].

\section{Evaluation of reactive oxygen species (ROS)}

To evaluate the oxidative stress in each group, the ROS level of each group was measured using CellROX Orange Reagent (Life Technologies) according to the manufacturer's instruction. Briefly, Brl-3A cells in each group were collected with trypsin, and washed with PBS for 
A

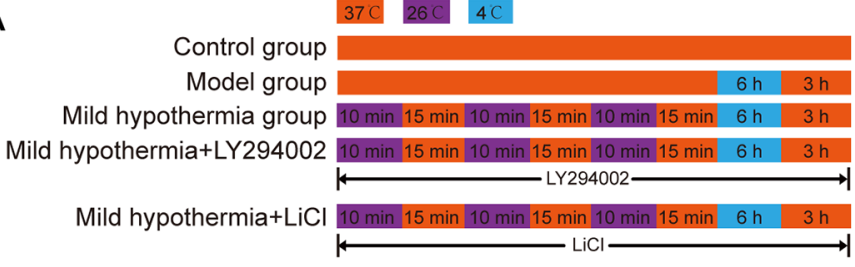

B

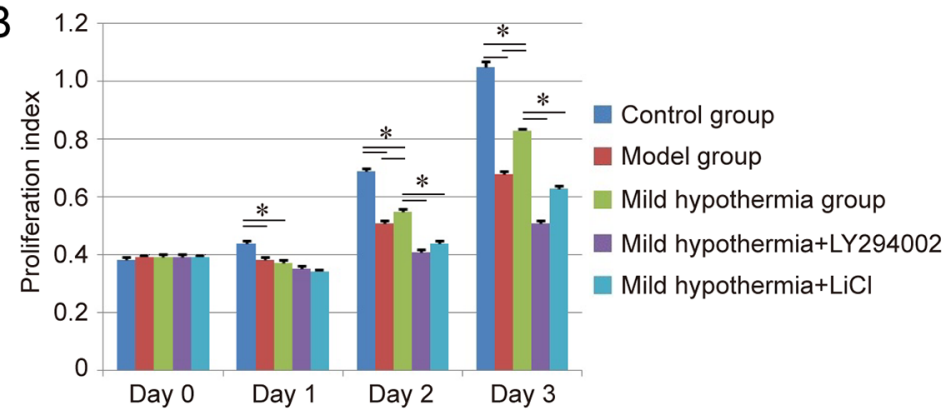

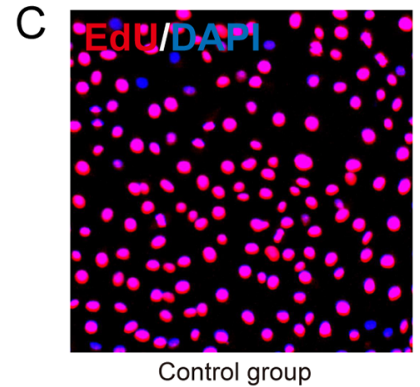

D 3.0 extracellular
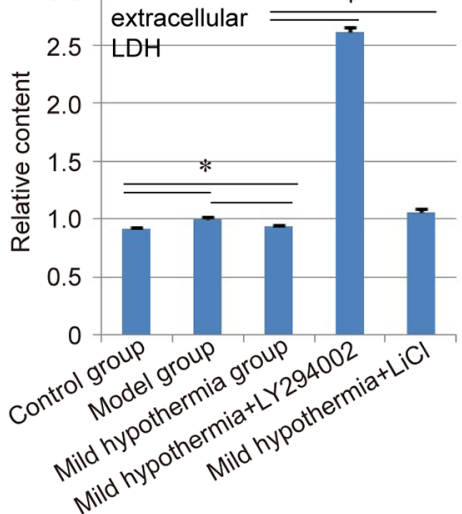

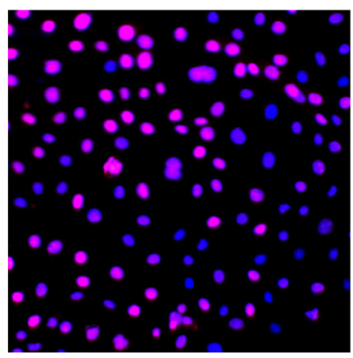

Model group

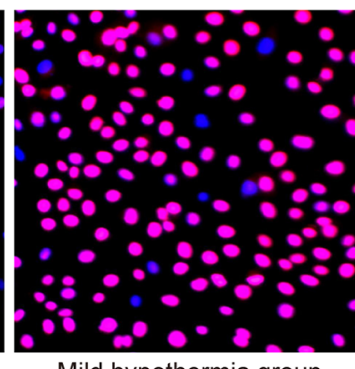

Mild hypothermia group

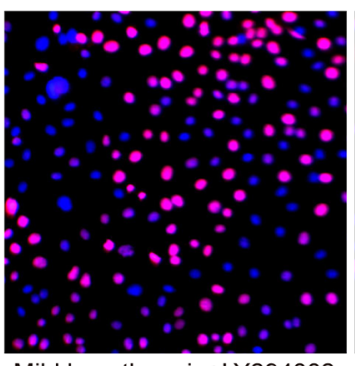

Mild hypothermia+LY294002

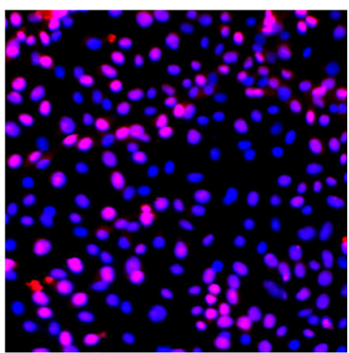

Mild hypothermia+LiCl
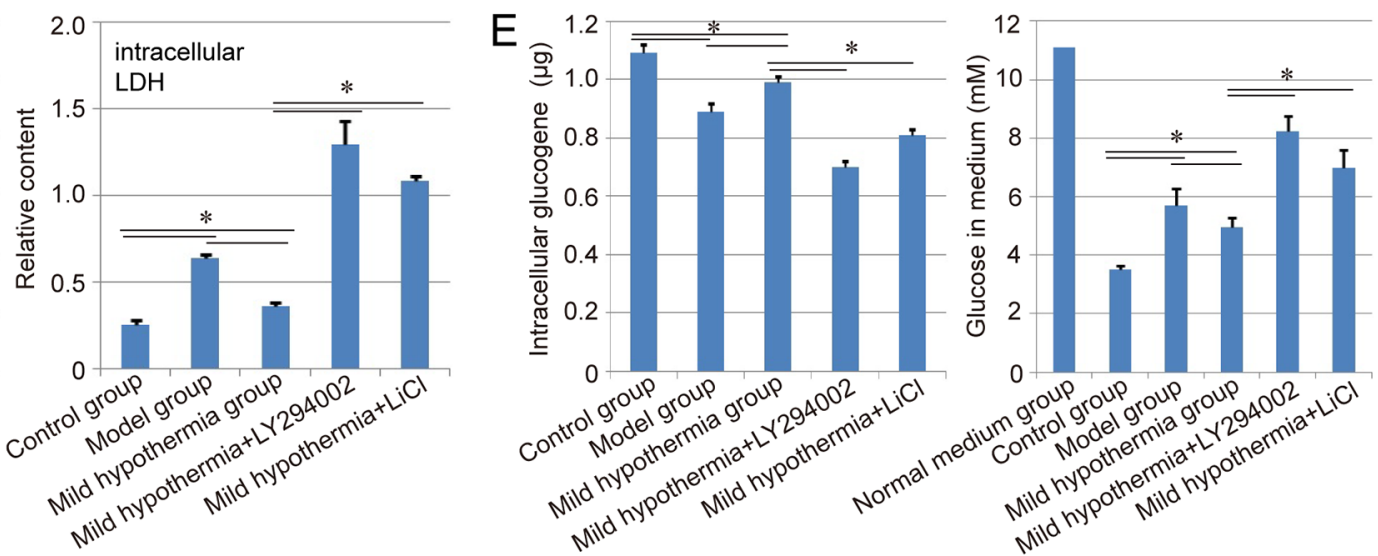

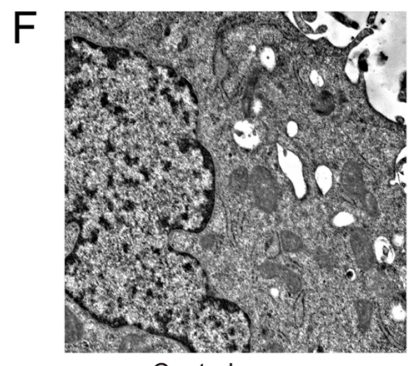

Control group

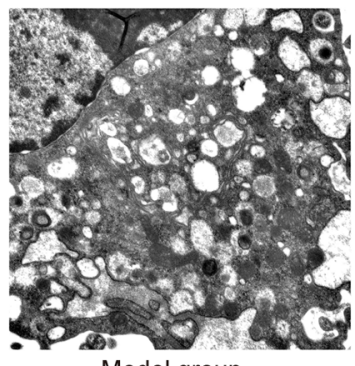

Model group

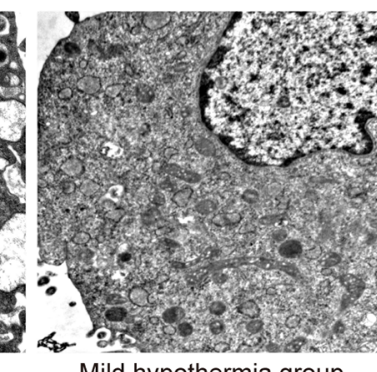

Mild hypothermia group

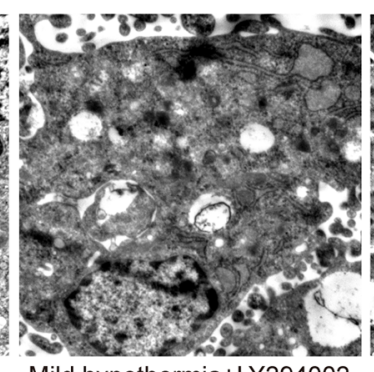

Mild hypothermia+LY294002

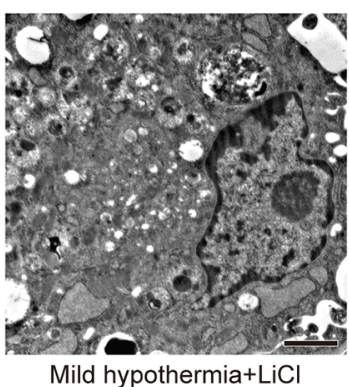

Fig 1. Effect of precondition with mild hypothermia on cell viability. A. Schematic diagram of the approach for treatment of each group. Red orange: the treatment with $37^{\circ} \mathrm{C}$; Purple: the treatment with $26^{\circ} \mathrm{C}$; Blue: the treatment with $4^{\circ} \mathrm{C}$. B-E. Evaluation of cell viability, cell proliferation ability, LDH regulation, glycogen synthesis ability and glucose absorption of each group respectively. Similar results were obtained in three independent experiments and results were expressed as mean \pm SEM. A t-test was used to compare the various groups, and $P<0.05$ was considered statistically significant. ${ }^{*}: P<0.05$ between the two groups. F. Ultrastructures of Brl-3A cells in each group. Scale bar corresponds to $1 \mu \mathrm{m}$.

https://doi.org/10.1371/journal.pone.0176652.g001

once, then the cells were further incubated with $50 \mu \mathrm{M}$ CellROX Reagent at $37^{\circ} \mathrm{C}$ for $30 \mathrm{~min}$, then washed with PBB for three times. The cells incubated with nothing were used as the negative group. Finally, the cell samples were analyzed using a FACSCalibur cytometer (BD). 


\title{
Cell apoptosis assay
}

In this study, cell apoptosis assay was performed using Vybrant apoptosis Assay Kit (Life Technologies) according to the manufacturer's instruction. In brief, Brl-3A cells in each group were dissociated into single cells with $0.25 \%$ trypsin, and further washed with PBS, then incubated with Hoechst 33342 and PI solution for $30 \mathrm{~min}$. The cells incubated with nothing were used as the negative group. Finally, the cell samples were analyzed using a FACSCalibur cytometer (BD).

\section{Evaluation of mitochondrial membrane potential (MMP) and mitochondrial membrane permeability transition pore (MPTP)}

JC-1 probe (Life Technologies) was used for the evaluation of MMP in each group. After the treatment of each group, the cells were washed with PBS, following by adding $1 \mathrm{ml}$ DMEM without FBS, then $1 \mathrm{ml} \mathrm{JC}-1$ solution $(10 \mu \mathrm{g} / \mathrm{mL})$ was added, and the system was further incubated at $37^{\circ} \mathrm{C}$ for $20 \mathrm{~min}$. The Images of each group were observed under a confocal laser scanning microscopy (Zeiss 710 NLO).

For the detection of MPTP in each group, Living Cell MPTP Fluorescence Detection kit (Genmed) was used in our study, and the operation was mainly performed according to the manufacturer's instruction. Finally, Samples of all groups were analyzed using a FACSCalibur cytometer (BD).

\section{Real-Time Quantitative PCR}

Total RNA was isolated using Trizol (Invitrogen) according to the manufacturer's instructions, followed by converting to cDNA using Reverse Transcription System (A3500, Promega). Then, the cDNA samples were used for Real-Time Quantitative PCR (qPCR) with SYBR Green qPCR SuperMix (Invitrogen) on the ABI PRISM 7500 Sequence Detection System. The relative amount of gene transcriptions was normalized to $\beta$-actin. The gene expression level of the normal group was regarded as " 1.0 ", and the relative expression level of the other groups was evaluated. All primer sequences ( $\left.5^{\prime}-3^{\prime}\right)$ are as follows:

\author{
caspase-3-F: 5' -AATTCAAGGGACGGGTCATG-3' \\ caspase-3-R: 5' -TGACACAATACACGGGATCTG-3' \\ caspase-9-F: 5' -CAACAACGTGAACTTCTGCC-3' \\ caspase-9-R: 5' -GTCAGGTCGTTCTTCACCTC-3' \\ PI3K -F: 5' -AGTCTGCAGGGACAAAGGAT-3' \\ PI3K -R: 5' -TGACATGCTGGTTTGAAAGC-3' \\ AKT -F: 5' -AACCGTGTCCTGCAGAACTC-3' \\ AKT-R: 5' -CACAATCTCCGCACCGTAGA-3'

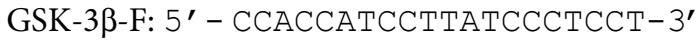 \\ GSK-3ß-R: 5' -GTTATTGGTCTGTCCACGGT-3' \\ ß-actin-F: 5' -AGGGAAATCGTGCGTGACAT-3' \\ $\beta$-actin-R: 5' -GAACCGCTCATTGCCGATAG-3'
}




\section{Western-blot}

The cell samples were harvested with RIPA lysis buffer and the protein content of cell lysates in each group was further determined using BCA protein estimation kit (Pierce, USA). Equal amounts $(20 \mathrm{mg})$ of protein were loaded per lane and electrophoresed in a $10 \%$ acrylamide gel $(120 \mathrm{~V}$ for $1 \mathrm{~h})$. The protein transfer was further performed using nitrocellulose for $1 \mathrm{~h}$ at 100 V. The primary antibodies used were anti-Cytochrome (1:500; Santa), anti-PI3K (1:400; Santa), anti-Akt (1:200; Santa), anti-p-Akt (1:200; Santa), anti-GSK-3 $\beta(1: 200$; Santa) and antip-GSK-3 $\beta$ (1:300; Santa). Anti-mouse, rabbit or goat HRP and an Amersham ECL kit (GE Healthcare) were further used to detect protein. The band densities were quantified by densitometry (Quantity One v4.62). GAPDH was used for normalization and the relative intensity level of the normal group was regarded as "1.0", and the relative intensity level of the other groups was evaluated.

\section{Immunofluorescence staining}

The cells were fixed in 4\% paraformaldehyde in PBS. For the Immunofluorescence staining, the primary antibodies used were anti-rat voltage dependent anion channel (VDAC; 1:100, Santa), anti-rat hexokinase 2 (HK2, 1:50, Santa). After 12 hours of incubation at $4^{\circ} \mathrm{C}$, the samples were washed 3 times with PBS and processed using second antibodies and DAPI, then observed using Axio Scope A1 as the reference [24, 25].

\section{Statistical analysis}

The results were presented as means \pm SEM, and the statistical analysis was performed with SPSS16.0. The differences among each group were analyzed by one-way ANOVA and followed by t-test. $P<0.05$ was considered statistically significant.

\section{Results}

\section{The pre-protective effect of mild hypothermia in liver cell injury model}

The viability of each group was evaluated with CCK- 8 method, and the results indicated that the control group hold best potential in cell proliferation compared with other groups. After culture for 2 days, we found that the mild hypothermia group showed better proliferation ability than the model group $(P<0.05)$, even though still worse than the control group. After adding LY294002 (PI3K inhibitor) or LiCl (GSK3 $\beta$ inhibitor) in the culture system, the viability of mild hypothermia group was further inhibited obviously $(P<0.05)$, indicating that PI3K-AktGSK3 $\beta$ signal pathway might play an important role in the pre-protective effect of mild hypothermia (Fig 1B). Besides, to evaluate the proliferation ability of each group, EdU assay was performed herein. The results indicated that nearly all of cells in the normal group showed positive staining of EdU, while the positive rate was reduced obviously in model group. The mild hypothermia preconditioning could enhance the positive rate significantly, indicating the protection of mild hypothermia from low temperature damage. Similar to CCK-8 result, the treatment of LY294002 or $\mathrm{LiCl}$ also could inhibit the pre-protective effect of mild hypothermia, suggesting the significance of PI3K-Akt-GSK3 $\beta$ signal pathway for the pre-protection (Fig $1 C)$.

To further evaluate the cell viability and function, the extracellular/intracellular LDH and intracellular glycogen in each group were measured in our study. Both extracellular LDH and intracellular LDH showed a similar tendency in each group, while the difference of the intracellular LDH among each group was more obvious than that of extracellular LDH. The model group hold higher level of LDH than the control group and mild hypothermia group $(P<0.05)$, 
indicating the pre-protective effect of mild hypothermia in the liver cell injury model (Fig 1D). On the other side, capability of glycogenesis was also inhibited in the model group, and the inhibition could be relieved by the precondition with mild hypothermia $(P<0.05)$. In addition, compared with mild hypothermia group, higher level of LDH and less glycogen could be detected in the groups of mild hypothermia + LY294002 and mild hypothermia $+\mathrm{LiCl}(P<0.05$, Fig $1 \mathrm{D}$ and $1 \mathrm{E})$. We also detected the glucose concentration of each group in medium, and the results indicated that the glucose concentration in each group was down-regulated compared with normal medium group. The mild hypothermia group showed better glucose absorption than the model group $(P<0.05$, Fig $1 \mathrm{E})$.

Cell samples from each group were further observed under TEM to evaluate the ultrastructures. The normal Brl-3A cells had clear ultrastructures with minimal heterochromatin and numerous organelles in the cytoplasm. In the model group, lots of vacuoles existed in the cytoplasm, as well as fewer organelles. This damage could be rescued through the precondition with mild hypothermia, and more endocytoplasmic reticulum and mitochondria could be observed in the mild hypothermia group, compared with model group. However, the rescue function also could be disturbed by LY294002 and LiCl in different degree (Fig 1F).

\section{ROS and cell apoptosis assay}

Scientists have indicated that hypothermia could protect hepatocytes from cell death through the reduction of ROS production in some liver disease [11]. Therefore, the ROS production in each group was evaluated in our study. In the FACS detection, the median of each group was used to evaluate the ROS production. Compared with control group (154.76 \pm 2.35 ), the higher level of ROS could be observed in the model group (196.44 \pm 6.57 ), and a little relief existed in the mild hypothermia group (178.53 \pm 6.42 ). As expected, the treatment of LY294002 and LiCl could further increase the ROS production in the damaged liver cells (Mild hypothermia+-

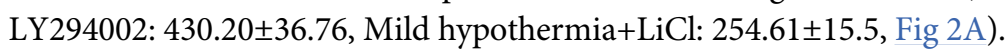

Besides, the cell apoptosis of each group was also analyzed through Hoechst 33342 and PI double staining. The staining assay revealed that low temperature-induced damage could lead to an increase in the percentage of early-stage apoptotic cells (Hoechst 33342-positive and PInegative cells), as well as late-stage apoptotic cells (Hoechst 33342-positive and PI-positive cells) in Brl-3A cells, and further analysis indicated that this apoptosis-induced effect could be inhibited with the precondition with mild hypothermia. Moreover, the groups of mild hypothermia + LY294002 and mild hypothermia + LiCl showed more apoptotic cells in both early stage and late stage than the mild hypothermia group (Fig 2B).

\section{Evaluation of mitochondrial mechanism}

To further analyze the pre-protective effect of mild hypothermia from liver cells damage on the level of subcellular organelle, both MMP and MPTP were analyzed in each group. Herein, JC-1 staining assay was carried out to determine the alteration of MMP. All of groups showed positive staining of JC-1 aggregates (red), while the staining of JC-1 monomers (green) was different in each group. Few JC-1 monomers could be observed in the control group, and the amount of JC-1 monomers was increased in the damaged liver cells. The mild hypothermia also exhibited the pre-protective effect and the number of cells with loss of mitochondrial membrane potential was decreased with the precondition (Fig 3A). Similar results were achieved in the MPTP detection with FACS, in which the median was also used to evaluate the MPTP in each group. Compared with control group (19.20 \pm 0.93$)$, the MPTP of the model group (25.80 \pm 1.21$)$ was increased in some degree. A little relief could be observed in the mild hypothermia group (22.41 \pm 0.78 ) (Fig 3B). In addition, LY294002 and $\mathrm{LiCl}$ could promote the 

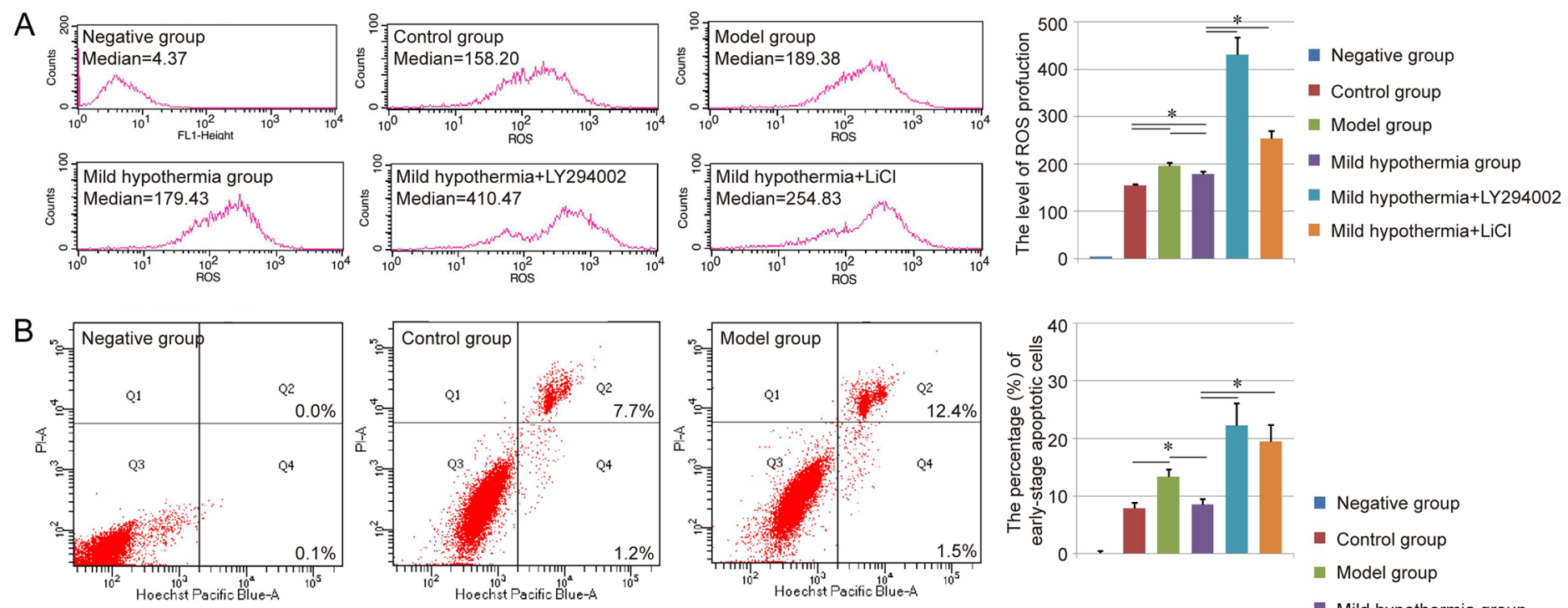

- Negative group

- Control group

- Model group

- Mild hypothermia group
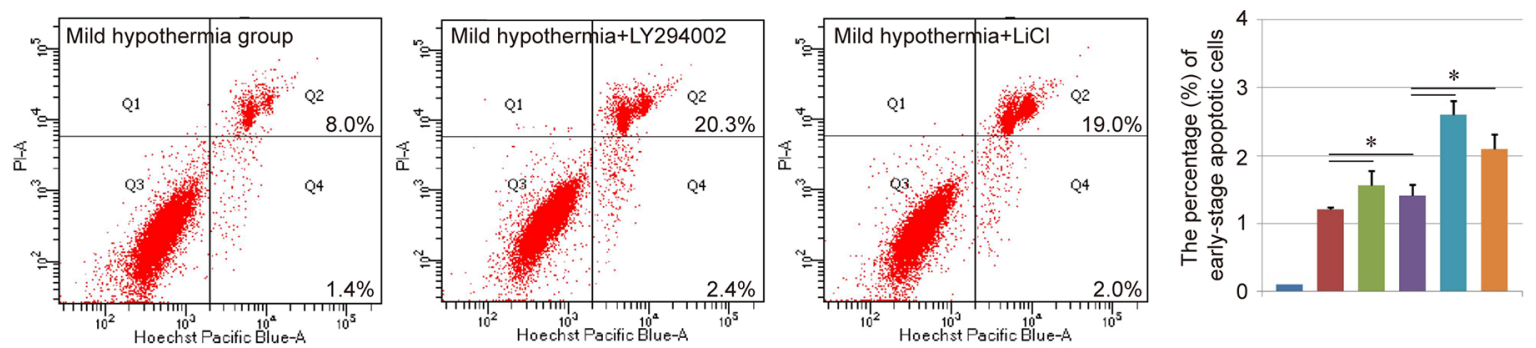

- Mild hypothermia+LY294002

Mild hypothermia+LiCl

Fig 2. ROS production and Cell apoptosis assay of each group. A. Evaluation of ROS production in each group with FACS. The median of each group was used to evaluate the ROS production. B. Cell apoptosis assay of each group via Hoechst 33342 and PI double staining. Both the early-stage apoptotic cells (Hoechst 33342-positive and PI-negative cells) and late-stage apoptotic cells (Hoechst 33342-positive and PI-positive cells) were analyzed in our study. Similar results were obtained in three independent experiments and results were expressed as mean \pm SEM. A t-test was used to compare the various groups, and $P<0.05$ was considered statistically significant. *: $P<0.05$ between the two groups.

loss of mitochondrial membrane potential in the detection of MMP and MPTP (Fig 3A and 3B). The cytochrome in mitochondria and kytoplasm was further detected respectively. We found that the amount of cytochrome in mitochondria and kytoplasm was reduced in the model group, compared with the control group and mild hypothermia group. However, amount of cytochrome in mitochondria and kytoplasm didn't show obvious difference between the control group and mild hypothermia group (Fig 3C). Besides, the expression of HK2 and VDAC, two kinds of mitochondrial membrane proteins, were evaluated with immunofluorescence in each group, and the results showed the down-regulation of HK2 and VDAC expression in the model group and up-regulation in the mild hypothermia group, indicating the relationship between the pre-protective effect of mild hypothermia and the regulation of mitochondrial membrane protein expression (Fig 3D).

We also detected the expression of Caspase 9, a gene associated with mitochondrial apoptotic pathway. The results indicated that the expression of Caspase 9 was up-regulated in the model group compared with the control group, and further down-regulated in the mild hypothermia group $(P<0.05$, Fig $3 \mathrm{E})$. Similar results could be found in the expression of Caspase 3 , another apoptotic gene, in each group (Fig 3E). With the treatment of LY294002 and LiCl, expression of the two apoptotic genes could be up-regulated obviously, indicating the function of PI3K-Akt-GSK3 $\beta$ signal pathway in the pre-protective effect of mild hypothermia (Fig 3E). Therefore, we further detected the expression of PI3K, Akt, and GSK3 $\beta$ in each group. The 

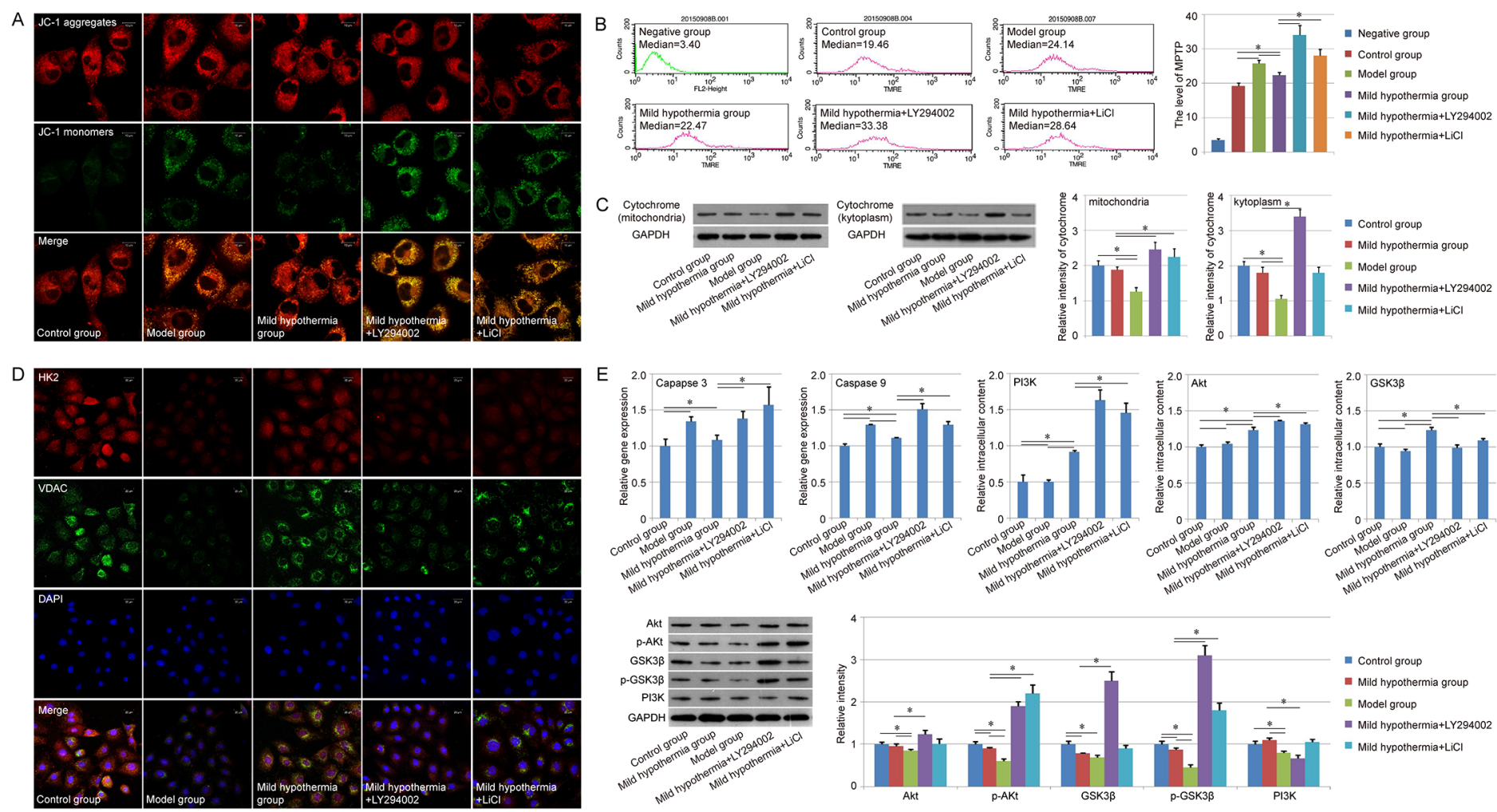

- Control group

Mild hypothermia group Model group

Mild hypothermia+LY294002 - Mild hypothermia+LiCl
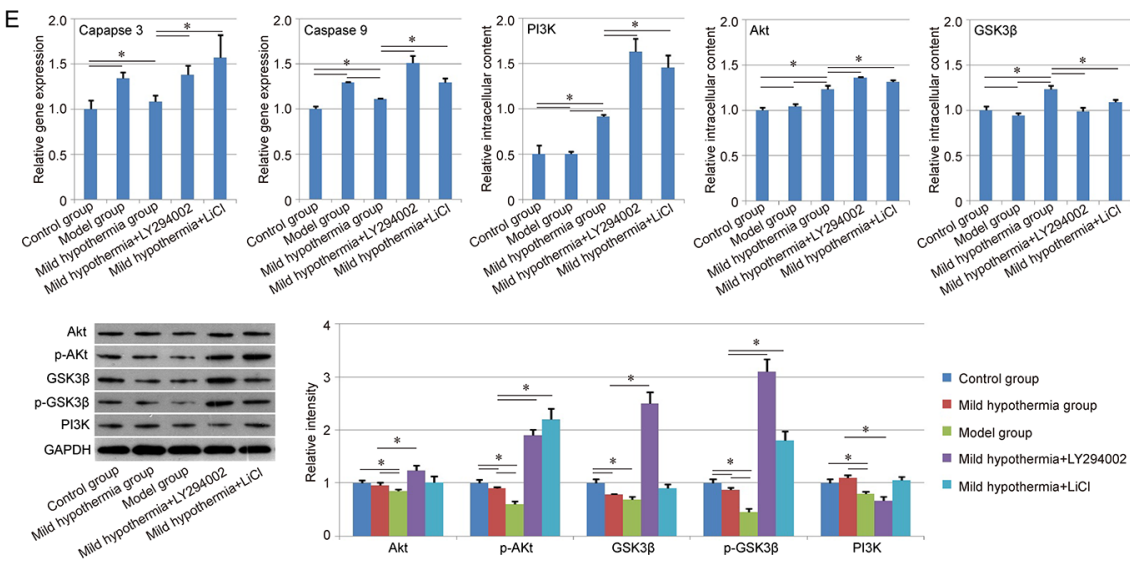

- Control group - Mild hypothermia group Model group - Mild hypothermia+LY294002 - Mild hypothermia+LiCl

Fig 3. Mitochondrial mechanism of the pre-protective effect of mild hypothermia. A. Evaluation of MMP of each group. Top: Staining of JC-1 aggregates (red); Middle: Staining of JC-1 monomers (green); Bottom: Marge of Top and Middle. Scale bar corresponds to $10 \mu \mathrm{m}$. B. Analysis of MPTP opening of each group with FACS. The median of each group was used to evaluate MPTP opening. C. Expression of cytochrome in mitochondria and kytoplasm. D. Evaluation of HK2 (red) and VDAC (green) expression with immunofluorescence. Scale bar corresponds to $20 \mu \mathrm{m}$. E. Gene expression assay with qPCR and western-blot. Similar results were obtained in three independent experiments and results were expressed as mean \pm SEM. A t-test was used to compare the various groups, and $P<0.05$ was considered statistically significant. *: $P<0.05$ between the two groups.

https://doi.org/10.1371/journal.pone.0176652.g003

qPCR results suggested that the precondition with mild hypothermia could increase the expression of those genes compared with model group $(P<0.05)$ (Fig 3E). In the level of protein, modest up-regulation of the three genes expression could be observed in the mild hypothermia group compared with model group. In addition, we also detected the functional proteins in PI3K-Akt-GSK3 $\beta$ signal pathway, p-Akt, and p-GSK3 $\beta$, and the result showed that the expression of p-Akt and p-GSK3 $\beta$ were decreased in the model group obviously, and the precondition with mild hypothermia also could increase the expression of p-Akt and pGSK3 $\beta$, suggesting that the pre-protective effect of mild hypothermia depended on the function of PI3K-Akt-GSK3 $\beta$ signal pathway in some degree (Fig 3E). With the treatment of inhibitors, $\mathrm{qPCR}$ results showed that the expression of GSK3 $\beta$ was down-regulated in the group of mild hypothermia + LY294002 and mild hypothermia $+\mathrm{LiCl}$, while the expression levels of PI3K and Akt were up-regulated in those groups. Western-blot results also indicated the upregulation of p-Akt and p-GSK3 $\beta$ in those groups (Fig 3E). This phenomenon might be due to the negative feedback control mechanisms in the culture system.

\section{Discussion}

Although several groups have indicated that mild hypothermia had a protective effect against liver cell injury, the pre-protective effect of hypothermia on the liver cell has not been determined so far [9-11]. In the present study, our results indicated that the precondition with mild 
hypothermia could inhibit ROS production and cell apoptosis and increase cell viability of Brl$3 \mathrm{~A}$ cell line, and develop a novel strategy for the storage of liver cell. Scientists have demonstrated that ROS are considered as a major mediator of inflammation and the reduction of ROS levels can lead to the attenuation of liver cell injury and apoptosis directly [26-30]. Therefore, the mild hypothermia group holds better ability on cell proliferation, LDH regulation and glycogen synthesis. However, Brl-3A cell line used in our study is different from primary hepatocype, which are always applied in clinic. For example, the Brl-3A cells hold strong proliferative ability and stable hepatic function, while primary hepatocype could only maintain their hepatic function and proliferative ability for a short period in vitro. Even though Brl-3A cell line is regarded as a good hepatic cell model and used to evaluate the liver cell function in vitro widely [31-34], the exploration with primary hepatocyte should hold more clinical significance. Our group isolated rat primary hepatocyte as the reference [35] and further analyzed the effect of hypothermia on cell viability and cell proliferation ability. Similar to Brl-3A cell line, our results indicated that precondition with mild hypothermia still hold protective function on rat primary hepatocyte (S1 Fig). However, the detailed mechanism about such phenomenon still need our further investigation.

Besides, we detected the temperature changing during the hypothermia preconditioning process (S2 Fig), and the result also indicated that $10 \mathrm{~min}$ or $15 \mathrm{~min}$ was enough for the heat exchange in our culture system. However, more than $10^{9}$ cells are needed for bioartificial liver normally [36-38]. Both the cell number and medium are much more than that used in our study. Therefore, the either $10 \mathrm{~min}$ or $15 \mathrm{~min}$ treatment indeed is not suitable for such largescale liver cell storage or real bioartificial liver. Therefore, the accurate time of mild hypothermia preconditioning still needs further preclinical exploration, even clinical study.

In addition, some scientists also indicated that the therapeutic mechanism of mild hypothermia may be related to mitochondrial protection. Mild hypothermia treatment could decrease MPTP opening and restore the MMP. Furthermore, hypothermia also could decrease mitochondrial malondialdehyde and elevate mitochondrial-reduced glutathione, consistent with the restored mitochondrial function [39]. However, the mechanisms about the reduction in MMP by mild hypothermia are complex [40]. For example, ischemia-reperfusion could increase the level of MPTP activators (Ca2p, Pi and ROS,) and reduce the level of MPTP inhibitors (ATP/ADP and low PH) [41]. Both the activator and inhibitors of MPTP opening could be impacted by the mild hypothermia in different degree, and the balance between the activator and inhibitors determined the duration of MPTP opening [39, 40, 42]. In this study, the reduction of MPTP opening and the restoration of MMP were also observed in the mild hypothermia group, as well as the inhibition of ROS production, a MPTP activator. The expression of Caspase 9, a gene associated with mitochondrial apoptotic pathway, was also down-regulated in the mild hypothermia group, compared with model group. Further detection showed that the expression of both HK2 and VDAC, two kinds of membrane proteins of mitochondria, could be down-regulated with low temperature treatment, and mild hypothermia preconditioning could up-regulated their expression in damaged hepatic cells. Thus, the preprotective effect of mild hypothermia should also be related to mitochondrial protection, and HK2 and VDAC might be the target proteins during the protection process. However, whether the detailed mechanism of MMP and MPTP regulation is similar to the previous reports, still needs our further exploration.

In our research, we found that the effect of precondition with mild hypothermia could be inhibited through adding LY294002 (PI3K inhibitor) or LiCl (GSK3 $\beta$ inhibitor) in the culture system. The results suggested that PI3K-Akt-GSK3 $\beta$ signal pathway might play an important role in the pre-protective effect of mild hypothermia. PI3K-Akt-GSK3 $\beta$ signal pathway has been demonstrated to be essential for the recovery of liver cell injury by some groups [43-47]. 
The pathway also has been indicated to hold the ability to regulate the protective effect of mild hypothermia against ischaemia/reperfusion injury. The expression levels of p-Akt, and pGSK3 $\beta$ in the mild hypothermia treated group were higher than those in the ischaemia/reperfusion group, and the therapeutic function also could be influenced by LY294002 [48, 49]. In our study, the results showed that the cell viability, MMP and other characteristics were much worse in the group of mild hypothermia + LY294002 and mild hypothermia + LiCl than those in the control group, the mild hypothermia group, even the model group, indicating that the PI3K-Akt-GSK3 $\beta$ signal pathway might be associated with not only pre-protective effect of mild hypothermia, but also other essential functions in liver cell. Interestingly, with the treatment of LY294002 and LiCl, the expression levels of some key genes in the pathway were upregulated in those groups. Maybe this phenomenon was due to the negative feedback control mechanisms in the low temperature-induced liver cell injury model. Anyway, this conclusion still needs our further confirmation. In addition, our study only evaluated the expression of PI3K, Akt/p-Akt and GSK3 $\beta / p$-GSK3 $\beta$ in the signal pathway with qPCR and western-blot preliminary, more detailed and accurate analysis about the pathway, such as the expression of upstream or downstream genes, should also be investigated intensively.

\section{Conclusion}

In conclusion, this study mainly investigated the pre-protective effect of mild hypothermia on low temperature-induced liver cell injury, and provided a novel strategy for the storage of liver cell in bioengineering. The results indicated that the precondition with mild hypothermia could enhance cell proliferation, LDH regulation and glycogen synthesis ability, and relief the ROS production and cell apoptosis in Brl-3A cells. Further analysis showed that the precondition also could decrease MPTP opening and restore MMP, and PI3K-Akt-GSK3 $\beta$ signal pathway might be associated with the pre-protective effect of mild hypothermia. However, the detailed mechanism of this effect still needs our further exploration.

\section{Supporting information}

S1 Fig. Effect of precondition with mild hypothermia on rat primary hepatocyte. A. Evaluation of cell viability with CCK-8. B. Analysis of cell proliferation ability with EDU staining. Similar results were obtained in three independent experiments and results were expressed as mean \pm SEM. A t-test was used to compare the various groups, and $P<0.05$ was considered statistically significant. *: $P<0.05$ between the two groups.

(PDF)

S2 Fig. The temperature changing during the hypothermia preconditioning process. (PDF)

\section{Author Contributions}

Conceptualization: JQ YG.

Data curation: JQ YM YG.

Formal analysis: ZJ YG.

Funding acquisition: YG.

Investigation: JQ YG.

Methodology: JQ YM YL ZJ. 
Project administration: JQ YG.

Resources: JQ YG.

Software: JQ YM.

Supervision: JQ YG.

Validation: JQ YM.

Visualization: JQ YG.

Writing - original draft: JQ YG.

Writing - review \& editing: JQ YG.

\section{References}

1. Puts CF, Berendsen TA, Bruinsma BG, Ozer S, Luitje M, Usta OB, et al. Polyethylene glycol protects primary hepatocytes during supercooling preservation. Cryobiology. 2015; 71(1):125-9. PubMed Central PMCID: PMC4506890. https://doi.org/10.1016/j.cryobiol.2015.04.010 PMID: 25936340

2. Ferrigno A, Rizzo V, Boncompagni E, Bianchi A, Gringeri E, Neri D, et al. Machine perfusion at 20 degrees $C$ reduces preservation damage to livers from non-heart beating donors. Cryobiology. 2011; 62(2):152-8. https://doi.org/10.1016/j.cryobiol.2011.02.004 PMID: 21315707

3. Jacobs SE. Selective head cooling with mild systemic hypothermia after neonatal encephalopathy: Multicentre randomised trial. The Journal of pediatrics. 2005; 147(1):122-3. https://doi.org/10.1016/j.jpeds. 2005.04.047 PMID: 16027712

4. Gluckman PD, Wyatt JS, Azzopardi D, Ballard R, Edwards AD, Ferriero DM, et al. Selective head cooling with mild systemic hypothermia after neonatal encephalopathy: multicentre randomised trial. Lancet. 2005; 365(9460):663-70. https://doi.org/10.1016/S0140-6736(05)17946-X PMID: 15721471

5. Wu TC, Grotta JC. Hypothermia for acute ischaemic stroke. The Lancet Neurology. 2013; 12(3):27584. https://doi.org/10.1016/S1474-4422(13)70013-9 PMID: 23415567

6. Azzopardi DV, Strohm B, Edwards AD, Dyet L, Halliday HL, Juszczak E, et al. Moderate hypothermia to treat perinatal asphyxial encephalopathy. The New England journal of medicine. 2009; 361(14):1349_ 58. https://doi.org/10.1056/NEJMoa0900854 PMID: 19797281

7. Gao XY, Huang JO, Hu YF, Gu Y, Zhu SZ, Huang KB, et al. Combination of mild hypothermia with neuroprotectants has greater neuroprotective effects during oxygen-glucose deprivation and reoxygenation-mediated neuronal injury. Scientific reports. 2014; 4:7091. https://doi.org/10.1038/srep07091 PMID: 25404538

8. Sabir H, Walloe L, Dingley J, Smit E, Liu X, Thoresen M. Combined Treatment of Xenon and Hypothermia in Newborn Rats—Additive or Synergistic Effect? PloS one. 2014; 9(10):e109845. https://doi.org/ 10.1371/journal.pone.0109845 PMID: 25286345

9. Blei A. Hypothermia for fulminant hepatic failure: a cool approach to a burning problem. Liver transplantation: official publication of the American Association for the Study of Liver Diseases and the International Liver Transplantation Society. 2000; 6(2):245-7.

10. Fu T, Blei AT, Takamura N, Lin T, Guo D, Li H, et al. Hypothermia inhibits Fas-mediated apoptosis of primary mouse hepatocytes in culture. Cell transplantation. 2004; 13(6):667-76. PMID: 15648737

11. Sakurai $T$, Kudo M, Watanabe $T$, Itoh $K$, Higashitsuji $H$, Arizumi T, et al. Hypothermia protects against fulminant hepatitis in mice by reducing reactive oxygen species production. Digestive diseases. 2013; 31(5-6):440-6. https://doi.org/10.1159/000355242 PMID: 24281018

12. Hong MF, Dorian P. Update on advanced life support and resuscitation techniques. Current opinion in cardiology. 2005; 20(1):1-6. PMID: 15596952

13. Diestel A, Roessler J, Berger F, Schmitt KR. Hypothermia downregulates inflammation but enhances IL-6 secretion by stimulated endothelial cells. Cryobiology. 2008; 57(3):216-22. https://doi.org/10.1016/ j.cryobiol.2008.08.005 PMID: 18790695

14. Sakurai T, Itoh K, Higashitsuji H, Nonoguchi K, Liu Y, Watanabe H, et al. Cirp protects against tumor necrosis factor-alpha-induced apoptosis via activation of extracellular signal-regulated kinase. Biochimica et biophysica acta. 2006; 1763(3):290-5. https://doi.org/10.1016/j.bbamcr.2006.02.007 PMID: 16569452 
15. Qadir XV, Chen W, Han C, Song K, Zhang J, Wu T. miR-223 Deficiency Protects against Fas-Induced Hepatocyte Apoptosis and Liver Injury through Targeting Insulin-Like Growth Factor 1 Receptor. The American journal of pathology. 2015; 185(12):3141-51. https://doi.org/10.1016/j.ajpath.2015.08.020 PMID: 26598234

16. Jacobs S, Jager S, Jansen E, Peter A, Stefan N, Boeing H, et al. Associations of Erythrocyte Fatty Acids in the De Novo Lipogenesis Pathway with Proxies of Liver Fat Accumulation in the EPIC-Potsdam Study. PloS one. 2015; 10(5):e0127368. PubMed Central PMCID: PMC4435749. https://doi.org/10. 1371/journal.pone.0127368 PMID: 25984792

17. Chen WN, Han C, Zhang JQ, Song K, Wang Y, Wu T. Deletion of Mir155 Prevents Fas-Induced Liver Injury through Up-Regulation of Mcl-1. American Journal Of Pathology. 2015; 185(4):1033-44. https:// doi.org/10.1016/j.ajpath.2014.12.020 PMID: 25794705

18. Tanoi T, Tamura $T$, Sano N, Nakayama K, Fukunaga K, Zheng YW, et al. Protecting liver sinusoidal endothelial cells suppresses apoptosis in acute liver damage. Hepatology research. 2016; 46(7):697706. https://doi.org/10.1111/hepr.12607 PMID: 26490536

19. Lian $F$, Wang $Y$, Xiao $Y, W u X, X u H$, Liang $L$, et al. Activated farnesoid $X$ receptor attenuates apoptosis and liver injury in autoimmune hepatitis. Molecular medicine reports. 2015; 12(4):5821-7. PubMed Central PMCID: PMC4581797. https://doi.org/10.3892/mmr.2015.4159 PMID: 26238153

20. Khaliulin I, Clarke SJ, Lin H, Parker J, Suleiman MS, Halestrap AP. Temperature preconditioning of isolated rat hearts-a potent cardioprotective mechanism involving a reduction in oxidative stress and inhibition of the mitochondrial permeability transition pore. The Journal of physiology. 2007; 581(Pt 3):1147-61. PubMed Central PMCID: PMC1976396. https://doi.org/10.1113/jphysiol.2007.130369 PMID: 17395631

21. Cai J, Zhang Y, Liu P, Chen S, Wu X, Sun Y, et al. Generation of tooth-like structures from integrationfree human urine induced pluripotent stem cells. Cell regeneration. 2013; 2(1):6. PubMed Central PMCID: PMC4230506. https://doi.org/10.1186/2045-9769-2-6 PMID: 25408878

22. Liu P, Feng Y, Dong C, Yang D, Li B, Chen X, et al. Administration of BMSCs with Muscone in Rats with Gentamicin-Induced AKI Improves Their Therapeutic Efficacy. PloS one. 2014; 9(5):e97123. https:// doi.org/10.1371/journal.pone.0097123 PMID: 24824427

23. Liu P, Feng Y, Dong C, Liu D, Wu X, Wu H, et al. Study on therapeutic action of bone marrow derived mesenchymal stem cell combined with vitamin E against acute kidney injury in rats. Life sciences. 2013; 92(14-16):829-37. https://doi.org/10.1016/j.lfs.2013.02.016 PMID: 23499556

24. Liu P, Chen S, Li X, Qin L, Huang K, Wang L, et al. Low immunogenicity of neural progenitor cells differentiated from induced pluripotent stem cells derived from less immunogenic somatic cells. PloS one. 2013; 8(7):e69617. PubMed Central PMCID: PMC3724937. https://doi.org/10.1371/journal.pone. 0069617 PMID: 23922758

25. Huang K, Liu P, Li X, Chen S, Wang L, Qin L, et al. Neural progenitor cells from human induced pluripotent stem cells generated less autogenous immune response. Science China Life sciences. 2014; 57 (2):162-70. https://doi.org/10.1007/s11427-013-4598-6 PMID: 24443177

26. Sakurai T, Kudo M, Umemura A, He G, Elsharkawy AM, Seki E, et al. p38alpha inhibits liver fibrogenesis and consequent hepatocarcinogenesis by curtailing accumulation of reactive oxygen species. Cancer research. 2013; 73(1):215-24. PubMed Central PMCID: PMC3605785. https://doi.org/10.1158/ 0008-5472.CAN-12-1602 PMID: 23271722

27. Xiao J, Lv Y, Lin B, Tipoe GL, Youdim MB, Xing F, et al. A novel antioxidant multitarget iron chelator M30 protects hepatocytes against ethanol-induced injury. Oxidative medicine and cellular longevity. 2015; 2015:607271. PubMed Central PMCID: PMC4334871. https://doi.org/10.1155/2015/607271 PMID: 25722794

28. Yu Y, Bai F, Liu Y, Yang Y, Yuan Q, Zou D, et al. Fibroblast growth factor (FGF21) protects mouse liver against $D$-galactose-induced oxidative stress and apoptosis via activating Nrf2 and PI3K/Akt pathways. Molecular and cellular biochemistry. 2015; 403(1-2):287-99. https://doi.org/10.1007/s11010-0152358-6 PMID: 25701356

29. Zhang F, Wang X, Qiu X, Wang J, Fang H, Wang Z, et al. The protective effect of esculentoside a on experimental acute liver injury in mice. PloS one. 2014; 9(11):e113107. PubMed Central PMCID: PMC4236201. https://doi.org/10.1371/journal.pone.0113107 PMID: 25405982

30. Hosseini MJ, Shahraki J, Tafreshian S, Salimi A, Kamalinejad M, Pourahmad J. Protective effects of Sesamum indicum extract against oxidative stress induced by vanadium on isolated rat hepatocytes. Environmental toxicology. 2016; 31(8):979-85. https://doi.org/10.1002/tox.22107 PMID: 25727928

31. Guo X, Cui R, Zhao J, Mo R, Peng L, Yan M. Corosolic acid protects hepatocytes against ethanolinduced damage by modulating mitogen-activated protein kinases and activating autophagy. European journal of pharmacology. 2016; 791:578-88. https://doi.org/10.1016/j.ejphar.2016.09.031 PMID: 27663281 
32. Seidah NG, Benjannet S, Wickham L, Marcinkiewicz J, Jasmin SB, Stifani S, et al. The secretory proprotein convertase neural apoptosis-regulated convertase 1 (NARC-1): liver regeneration and neuronal differentiation. Proceedings of the National Academy of Sciences of the United States of America. 2003; 100(3):928-33. PubMed Central PMCID: PMC298703. https://doi.org/10.1073/pnas. 0335507100 PMID: 12552133

33. Zuo D, Duan Z, Jia Y, Chu T, He Q, Yuan J, et al. Amphipathic silica nanoparticles induce cytotoxicity through oxidative stress mediated and p53 dependent apoptosis pathway in human liver cell line HL7702 and rat liver cell line BRL-3A. Colloids and surfaces B, Biointerfaces. 2016; 145:232-40. https:// doi.org/10.1016/j.colsurfb.2016.05.006 PMID: 27187187

34. Pan C, Chen H, Wang L, Yang S, Fu H, Zheng Y, et al. Down-regulation of MiR-127 facilitates hepatocyte proliferation during rat liver regeneration. PloS one. 2012; 7(6):e39151. PubMed Central PMCID: PMC3376093. https://doi.org/10.1371/journal.pone.0039151 PMID: 22720056

35. Takahara I, Akazawa Y, Tabuchi M, Matsuda K, Miyaaki H, Kido Y, et al. Toyocamycin attenuates free fatty acid-induced hepatic steatosis and apoptosis in cultured hepatocytes and ameliorates nonalcoholic fatty liver disease in mice. PloS one. 2017; 12(3):e0170591. https://doi.org/10.1371/journal.pone. 0170591 PMID: 28278289

36. Enosawa S, Miyashita T, Saito T, Omasa T, Matsumura T. The significant improvement of survival times and pathological parameters by bioartificial liver with recombinant HepG2 in porcine liver failure model. Cell transplantation. 2006; 15(10):873-80. PMID: 17299991

37. Selden C, Spearman CW, Kahn D, Miller M, Figaji A, Erro E, et al. Evaluation of encapsulated liver cell spheroids in a fluidised-bed bioartificial liver for treatment of ischaemic acute liver failure in pigs in a translational setting. PloS one. 2013; 8(12):e82312. PubMed Central PMCID: PMC3867376. https:// doi.org/10.1371/journal.pone.0082312 PMID: 24367515

38. Enosawa S, Miyashita T, Fujita Y, Suzuki S, Amemiya H, Omasa T, et al. In vivo estimation of bioartificial liver with recombinant HepG2 cells using pigs with ischemic liver failure. Cell transplantation. 2001; 10(4-5):429-33. PMID: 11549067

39. Xiong W, Xu S, Li Md H, Liang K. Moderate hypothermia ameliorates enterocyte mitochondrial dysfunction in severe shock and reperfusion. The Journal of surgical research. 2016; 200(1):250-9. https://doi. org/10.1016/j.jss.2015.06.068 PMID: 26227675

40. Gong P, Hua R, Zhang Y, Zhao H, Tang Z, Mei X, et al. Hypothermia-induced neuroprotection is associated with reduced mitochondrial membrane permeability in a swine model of cardiac arrest. Journal of cerebral blood flow and metabolism: official journal of the International Society of Cerebral Blood Flow and Metabolism. 2013; 33(6):928-34. PubMed Central PMCID: PMC3677114.

41. Baines $C P$. The mitochondrial permeability transition pore and ischemia-reperfusion injury. Basic research in cardiology. 2009; 104(2):181-8. PubMed Central PMCID: PMC2671061. https://doi.org/10. 1007/s00395-009-0004-8 PMID: 19242640

42. Petronilli V, Penzo D, Scorrano L, Bernardi P, Di Lisa F. The mitochondrial permeability transition, release of cytochrome $c$ and cell death. Correlation with the duration of pore openings in situ. The Journal of biological chemistry. 2001; 276(15):12030-4. https://doi.org/10.1074/jbc.M010604200 PMID: 11134038

43. Lai SS, Zhao DD, Cao P, Lu K, Luo OY, Chen WB, et al. PP2Acalpha positively regulates the termination of liver regeneration in mice through the AKT/GSK3beta/Cyclin D1 pathway. Journal of hepatology. 2016; 64(2):352-60. https://doi.org/10.1016/j.jhep.2015.09.025 PMID: 26456844

44. Zheng T, Yang X, Wu D, Xing S, Bian F, Li W, et al. Salidroside ameliorates insulin resistance through activation of a mitochondria-associated AMPK/PI3K/Akt/GSK3beta pathway. British journal of pharmacology. 2015; 172(13):3284-301. PubMed Central PMCID: PMC4500366. https://doi.org/10.1111/bph. 13120 PMID: 25754463

45. Kim HJ, Joe Y, Kong JS, Jeong SO, Cho GJ, Ryter SW, et al. Carbon monoxide protects against hepatic ischemia/reperfusion injury via ROS-dependent Akt signaling and inhibition of glycogen synthase kinase 3beta. Oxidative medicine and cellular longevity. 2013; 2013:306421. PubMed Central PMCID: PMC3880761. https://doi.org/10.1155/2013/306421 PMID: 24454979

46. Lakshmanan J, Zhang B, Nweze IC, Du Y, Harbrecht BG. Glycogen synthase kinase 3 regulates IL1 beta mediated iNOS expression in hepatocytes by down-regulating c-Jun. Journal of cellular biochemistry. 2015; 116(1):133-41. https://doi.org/10.1002/jcb.24951 PMID: 25160751

47. Iqbal J, McRae S, Banaudha K, Mai T, Waris G. Mechanism of hepatitis C virus (HCV)-induced osteopontin and its role in epithelial to mesenchymal transition of hepatocytes. The Journal of biological chemistry. 2013; 288(52):36994-7009. PubMed Central PMCID: PMC3873557. https://doi.org/10. 1074/jbc.M113.492314 PMID: 24240095

48. Dai HB, Xu MM, Lv J, Ji XJ, Zhu SH, Ma RM, et al. Mild Hypothermia Combined with Hydrogen Sulfide Treatment During Resuscitation Reduces Hippocampal Neuron Apoptosis Via NR2A, NR2B, and PI3K- 
Akt Signaling in a Rat Model of Cerebral Ischemia-Reperfusion Injury. Molecular neurobiology. 2016; 53(7):4865-73. https://doi.org/10.1007/s12035-015-9391-z PMID: 26350917

49. Mochizuki T, Yu S, Katoh T, Aoki K, Sato S. Cardioprotective effect of therapeutic hypothermia at 34 degrees $\mathrm{C}$ against ischaemia/reperfusion injury mediated by $\mathrm{PI} 3 \mathrm{~K}$ and nitric oxide in a rat isolated heart model. Resuscitation. 2012; 83(2):238-42. https://doi.org/10.1016/j.resuscitation.2011.08.013 PMID: 21875500 